\title{
DECISION SUPPORT SYSTEM WITH SIMULATION-BASED OPTIMIZATION FOR HEALTHCARE CAPACITY PLANNING
}

\author{
Canan Gunes Corlu \\ John Maleyeff \\ Chenshu Yang \\ Tianhuai Ma \\ Yanting Shen \\ Metropolitan College \\ Boston University \\ 1010 Commonwealth Avenue \\ Boston, MA, 02215 USA
}

\begin{abstract}
Capacity management of hospital staff and other resources is an important challenge faced by a healthcare administrator. Because of the variation in service times and the inability to inventory services, capacity buffers are required to ensure reasonable waiting times for patients. The nonlinear relationship between resource utilization and patient wait times makes it difficult to determine the optimal capacity buffer, called the knee. This work concerns the development of a decision support system using Python to determine optimal capacity buffers using a Monte Carlo simulation and knee optimization model that allows for flexibility in specifying uncertain arrival patterns and service times. Key factors relating to the system's size, amount of service time variation, and arrival patterns are shown to affect optimal buffer sizes. The system shows users their current status and where changes need to be made to the service times or the number of servers to achieve optimal results.
\end{abstract}

\section{Keywords:}

simulation, optimization, healthcare, capacity planning

\section{INTRODUCTION}

When asked why he engaged in research on the mathematics of queues, John D.C. Little, the famed operations research pioneer and MIT professor, stated that one day he discovered "queues are everywhere." Indeed, all healthcare professionals are well aware of queues - usually in the form of patients waiting in a hospital's emergency department (ED), their physician's office, or at a pharmacy. In fact, there is potential for a queue associated with every resource a healthcare system employs to serve patients. In an MRI facility, for example, there may be a queue for parking, a queue for the elevator, a queue for checking in, a queue for the device, a queue for the technician, a queue for image interpretation, and a queue for payment. Other queues are hidden from obvious view, such as physicians waiting for blood test results, patients waiting for a call from their physician, or others waiting on hold for a call center representative.

When planning capacity, healthcare administrators may allocate resources to processes at levels somewhat higher than the demand forecast in order to provide effective customer service. Although Little's Law (which states that the average waiting time is equal to the ratio of the number of customers in queue and the service rate) is known to many practitioners, they do not always appreciate the non-linear relationship between server utilization (the average percentage of time spent serving customers) and customer wait times. Figure 1 shows a generic example that is applicable to any queuing system. As the server utilization increases, waiting time will increase in a pattern commonly known as a hockey stick. 


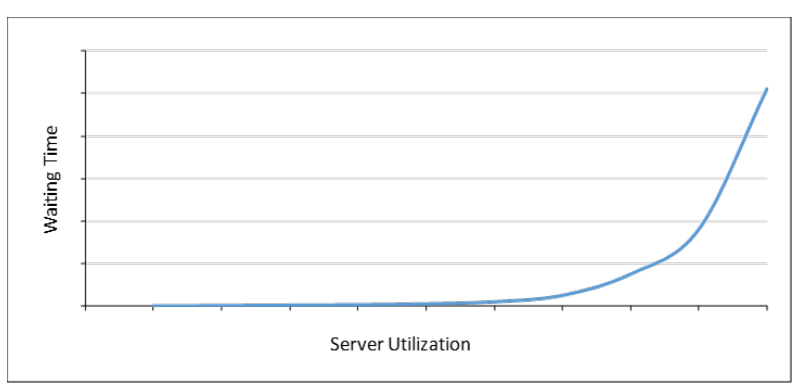

Figure 1: Server Utilization versus Waiting Time "Hockey-Stick."

The hockey-stick phenomenon has great implications for planners who wish to most effectively utilize their resources. Ideally, a capacity plan will effectively balance the needs of the planner (i.e., by maximizing server utilization) and the needs of the patient (by minimizing waiting times). In some fields, most notable computer science, the optimal system configuration takes place at a threshold referred to as the knee of the curve. Visually, the knee would be positioned at the point just before the slope of the hockey-stick curve significantly increases. The knee represents the optimal server utilization and capacity buffer (i.e., a $92 \%$ knee corresponds to a $8 \%$ capacity buffer). The goal of this paper is to propose a decision support system (DSS) for use by capacity planners to identify the location of the knee. The targeted application would be any multitude of queues found in healthcare settings. Queuing theory has been used to analyze queuing systems, but the restrictive assumptions associated with well-known queuing models preclude its effective use in many real-world settings.

This paper is organized as follows. Literature pertaining to healthcare capacity planning and queuing theory is reviewed, along with the challenges of embedding a Monte Carlo simulation (MCS) into a DSS. The methodology is then described, including the approach to finding the optimal capacity buffer (i.e., the knee). Then, the simulation model is described and used to evaluate a wide range of queues that could represent most queuing systems encountered by a healthcare administrator. Important results are discussed focusing on buffer recommendations for systems with various sizes and patterns of variation. Finally, the development and use of the DSS is described.

\section{LITERATURE SEARCH}

Congested systems (i.e., those with over-utilized servers and long customer wait times) have a negative impact on profit and customer satisfaction in many industries, including restaurants (Jain and Ali 2016), call centers (Sze 1984), and hospitals (Camacho et al. 2006). Medical professionals experience stress that leads to lower efficiency in congested systems (Sze 1984). Patients' expectations also increase in proportion to the time spent waiting for a service (Grossman 1972). And, health conditions of patients deteriorate as waiting times increase (Schulz 2017).

In healthcare, capacity buffering has been employed to hedge against congestion when the number of patients increases (Terwiesch et al. 2011; Towers 2014). The balancing of factors when determining capacity buffers in light of the high costs of healthcare delivery has been discussed (Bittencourt et al. 2018). Creating capacity buffers can be achieved by adding more resources or by reducing service times. Service times can be decreased by incorporating new technologies or removing non-value-added activities from the service process (Nicolaou 2016).

The use of queuing theory in healthcare capacity planning is relatively recent (Vass and Szabo 2015; Patel 2015; Gonzalez-Horta et al. 2011). Queuing theory has been applied frequently in the emergency department (ED) where both patient arrivals and treatment times are subject to uncertainty (Laskowski et al. 2009; Wang et al. 2013). In fact, two of the top three challenges faced by ED managers are shortage of inpatient beds and long patient flow times (Statista 2016). MCS is used when queues are complex (Lee and Elcan 1996). For example, it has helped healthcare managers make better capital decisions (Kennedy 2009). The simulation approach is robust because it allows a user to change many parameters (Zilm et al. 2003) including setting up work shifts (Kang and Park 2015). Physical resources are important to consider, such as beds, because they are often the bottleneck resource in the ED (Schiff 2011). 
A DSS can assist healthcare administrators by providing a means to implement consistent and reliable recommendations (Marcial et al. 2018). Because a DSS may be active (i.e., it makes the decision) or passive (i.e., it evaluates a decision), it can impact how administrators make decisions (Berner and La Lande 2007). The DSS should include pertinent information, a user interface, a mathematical or empirical model, and optimization criteria (Spooner 2016). It is especially valuable when it provides a mechanism to treat seemingly-different but similar problems using a structured approach that avoids having to reinvent the wheel (Koutsoukis and Mitra 2003). It works best when the DSS is developed by an integrated team of developers, practitioners, and users (Yasnoff and Miller 2003).

Models embedded in a DSS can take many forms, from qualitative rules-based checklists to artificial intelligence methodologies (Reyna et al. 2015). Stochastic (i.e., probability-based) decision models have been employed (Maleyeff et al. 2004), including those that address designing a clinical testing system (Benneyan and Kaminsky 1996) and those that evaluate images with signal detection models (Lynn and Barrett 2014). Kadri et al. (2014) use a model that simulates transition between various states of the ED. Embedding a MCS into a DSS can be difficult because simulations tend to be case specific and therefore cannot always be relied upon to apply in more general settings (Hertz et al. 2014). When using this approach, the system developer needs to be cognizant that output will vary randomly and therefore the DSS must include a proper balance of accuracy and execution time (Fanti et al. 2015). Some developers address output uncertainty indirectly by requiring users of the DSS to input the number of iterations (Yu et al. 2019). The contribution represented by the application presented here seeks to determine the optimal capacity buffer based on specific parameters associated with a healthcare queuing system.

\section{METHODOLOGY}

The queuing system assumed here is robust with the following structure: (1) customers wait in a single queue to be served by multiple parallel servers, (2) the population of customers is infinite, (3) customers arrive according to an assumed deterministic or random pattern, (4) there is no limit to the queue size, (5) the queue employs a first-come first-served discipline, and (6) service time follows an assumed random pattern. The assumptions regarding customer arrivals enables the system to assume that appointments are made (deterministic, or scheduled arrivals) or that they arrive according to a Poisson process (random arrivals). The Poisson assumption is valid because customers mainly arrive independently of one another.

For the analysis below, service time distributions are right-skewed because most atypical patients require longer service times than typical patients. Treatment times in healthcare often follow similar patterns that can be effectively modeled with the gamma distribution (Millhiser and Veral 2019). The gamma distribution is right skewed and flexible based on the coefficient of variation $(\mathrm{CV})$ of the service times. Figure 2 shows gamma distributions for various values of the CV. Its skewness is directly proportional to the $\mathrm{CV}$ (when the $\mathrm{CV}=1.0$, it is known as exponential distribution). In practice, the service time $\mathrm{CV}$ needs to be determined from the data collected at the facilities being modeled. The simulation described below can be easily adapted to other service time distributions.

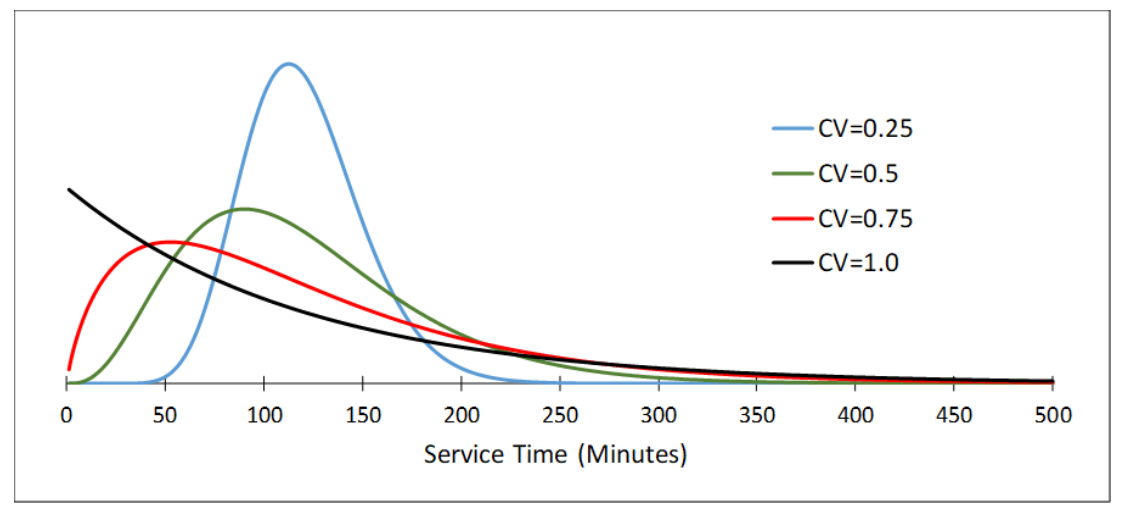

Figure 2: Gamma Distributions (Average Service Time is 120 Minutes). 
The performance of any queuing system is determined by the server utilization $(\rho)$, which is the ratio of the customer arrival rate $(\lambda)$ to the system's service rate. The system's service rate is the product of the number of servers $(s)$ and the service rate for each server $(\mu)$. Typically, performance is measured based on the time spent by customers in the system, the customer's wait time, the number of customers in the system, and the length of the queue.

\subsection{Simulation Model}

The MCS and knee-optimization algorithm were developed in Python, a programming language that is offered free of charge by the Python Software Foundation. The simulation logic mimics the queuing system by routing patients through a facility while keeping track of waiting times, total time spent in the system, and queue sizes. The inputs are the value of $s$, the $\mathrm{CV}$ of the gamma service time distribution, and the arrival pattern (scheduled or random). The MCS standardizes the queuing system to generate performance statistics based on the value of $\rho$. The model is applicable in a wide-range of applications without the need for re-programming the Python code.

The results obtained from the MCS were validated by using the analytical formulas of the M/M/s model ( $s$ parallel servers with exponential service times and Poisson arrivals). The MCS is run for a specified number of iterations, and a specified number of initial and terminal iterations dropped from consideration when calculating performance statistics. When employing a MCS, users need to be aware that output of the simulation will vary randomly. Therefore, the simulation is run for a specified number of macro-replications so that a statistical confidence interval (CI) can be calculated for each key result. The length of the CI is inversely proportional to the number of iterations and macro-replications, with run times taken into consideration for practical reasons.

\subsection{Optimization Model}

The knee (optimal server utilization) is employed by computer scientists to control network congestion. A popular approach was developed by Kleinrock (2018) who used a power function that identifies the level of server utilization that maximizes its good (i.e., server utilization) as compared to its bad (customer time in system). At various points of the hockey-stick function, power is calculated as the ratio of good to bad, using the following equation (where $W_{s}$ is the average time a customer spends in the system):

$$
P(G)=\frac{\rho}{\mu W_{s}}
$$

In the power function, the numerator is equal to the server utilization and the denominator is equal to the normalized average time a customer spends in the system. It is normalized to have a minimum value of 1.0 for any combination of inputs and will fall in the range of 1.0 to 2.0 when the power is maximized. Therefore, it provides an unbiased scaling for power function calculation. The MCS finds the knee (optimal server utilization) by systematically changing the value of $\rho$ from $40 \%$ to $95 \%$ (in increments of 5\%) and simulating the system repeatedly over this range. This approach generates the information required to quantify the hockey-stick graph.

\section{NOTEWORTHY OBSERVATIONS}

A factorial experimental design was used to explore how the optimal server utilization (i.e., the knee) changed based on various levels of key variables. The MCS was run for 88 combinations of factors, corresponding to: (a) two levels of arrivals (scheduled or random); (b) four levels of service time CV $(0.25,0.5,0.75$, or 1$)$; and (c) 11 levels for the number of servers. When determining the knee for each condition, 10 trials with 10000 iterations each were used to generate results for every level of server utilization. The 10000 iterations at each trial are extracted from the middle of 12000 runs.

\subsection{Congestion and Instability}

Although it is obvious that systems with higher server utilization exhibit longer wait times, the MCS showed that when the system is congested: (a) the variation of wait times increases as a percentage of the average wait time, and (b) wait times behaved erratically. Figure 3 shows the 95\% CI for the 
average wait time when 15 servers are employed, 6 customers per hour arrive randomly, and the service time CV is $50 \%$. When $\rho=0.80$ the average wait time was 8.6 minutes and its standard error was 0.29 minutes (a ratio of 3.4\%). When $\rho=0.95$ the average wait time was 94.7 minutes and its standard error was 8.93 minutes (a ratio of $9.4 \%$ ).

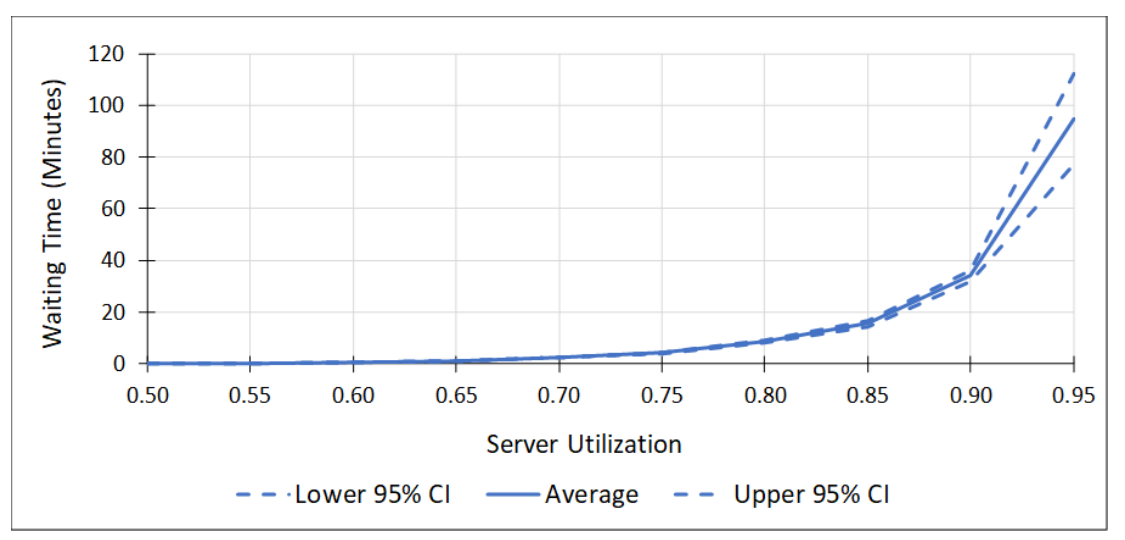

Figure 3: Example Confidence Intervals ( $\mathrm{s}=15, \mathrm{CV}=0.5,6 /$ hour random arrivals).

Although not shown here, the variation of the time spent in a congested system (e.g., $\rho=0.95$ ) exhibited a great deal of instability due to the significant autocorrelation among patient wait times. The impact of autocorrelation across patients was much less evident when $\rho=0.80$. There are implications for managers who would typically be unaware of the instability resulting from congested queuing systems. They may resort to tampering, which occurs when changes are made to a system in response to random variation, often causing the system to operate less effectively and impacting worker morale (Deming 1986, p. 327).

\subsection{Relieving Congestion}

The most obvious approach to reducing wait times would be the addition of servers. Capacity buffers would increase by hiring more staff or by placing clinical personnel "on call," both of which have financial implications. A less costly approach is to reduce service times. As an example, consider a system with 15 servers, where the arrival rate is 6 patients per hour and the service time CV is $50 \%$. In this case, an average service time of 142.5 minutes results in $\rho=95 \%$. As shown in Figure 3, the average wait time is 94.7 minutes. If the average service time can be reduced from 142.5 minutes to 127.5 minutes (now $\rho=85 \%$ ), the average wait time decreases to 15.5 minutes. This is a somewhat counter-intuitive result for many practitioners that is consistent with the hockey-stick function. That is, a 15 minute reduction of average service times will reduce wait times by an average of 79.2 minutes.

The ability to reduce service times is situation-dependant. In an ED, for example, patients occupy a bed while they wait for a physician, various treatments, or to be discharged. In many cases, a significant portion of this time is not value-added. Consider the process used to test blood when ordered by a physician. The process, including drawing the blood, testing the blood, and evaluating the results, may take as little as 15-20 minutes. But, the total processing time can be several hours due to waiting for the technician to draw the blood, moving the blood to the lab, waiting for previous tests to be completed, setting up the testing equipment, entering the patient's information, waiting for a volunteer to transport the blood to the lab, and waiting for the physician to complete other activities before viewing the results. At one hospital, the service time was reduced by creating a process whereby the lab was notified when the blood testing order was written (rather than when the blood arrived) so that they could plan an efficient testing sequence.

\subsection{Optimal Capacity Buffering}

Table 1 shows optimal server utilization (i.e., the knee) for the 88 conditions evaluated with the MCS. As expected, the value of the knee is inversely proportional to the levels of service time variation. That is, the knee is higher for scheduled arrivals (which exhibit less variation than random arrivals), and the knee is higher for lower service time CV values. 
Corlu, Maleyeff, Yang, Ma, and Shen

\begin{tabular}{|c|c|c|c|c|c|c|c|c|c|}
\hline & \multirow[b]{3}{*}{ CV } & \multicolumn{8}{|c|}{ Queuing System Assumptions } \\
\hline & & \multicolumn{4}{|c|}{ Random Arrivals } & \multicolumn{4}{|c|}{ Scheduled Arrivals } \\
\hline & & $25 \%$ & $50 \%$ & $75 \%$ & $100 \%$ & $25 \%$ & $50 \%$ & $75 \%$ & $100 \%$ \\
\hline \multirow{11}{*}{ 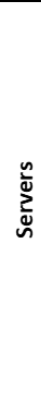 } & 1 & 0.60 & 0.55 & 0.50 & 0.50 & 0.85 & 0.75 & 0.65 & 0.55 \\
\hline & 2 & 0.65 & 0.65 & 0.60 & 0.60 & 0.90 & 0.80 & 0.70 & 0.65 \\
\hline & 3 & 0.70 & 0.70 & 0.70 & 0.60 & 0.90 & 0.85 & 0.80 & 0.75 \\
\hline & 4 & 0.70 & 0.75 & 0.70 & 0.65 & 0.90 & 0.85 & 0.80 & 0.75 \\
\hline & 5 & 0.75 & 0.75 & 0.70 & 0.65 & 0.90 & 0.85 & 0.80 & 0.75 \\
\hline & 10 & 0.80 & 0.80 & 0.80 & 0.75 & 0.95 & 0.90 & 0.85 & 0.85 \\
\hline & 15 & 0.85 & 0.80 & 0.85 & 0.80 & 0.95 & 0.90 & 0.90 & 0.90 \\
\hline & 25 & 0.85 & 0.85 & 0.85 & 0.85 & 0.95 & 0.95 & 0.90 & 0.90 \\
\hline & 50 & 0.90 & 0.90 & 0.90 & 0.90 & 0.95 & 0.95 & 0.95 & 0.90 \\
\hline & 75 & 0.90 & 0.90 & 0.90 & 0.95 & 0.95 & 0.95 & 0.95 & 0.95 \\
\hline & 100 & 0.95 & 0.95 & 0.90 & 0.95 & 0.95 & 0.95 & 0.95 & 0.95 \\
\hline
\end{tabular}

Table 1: Optimal Knee Values.

Figure 4 illustrates how the Power function is used to determine the knee for 4 of the 88 experimental combinations. In this case, the knee is $55 \%(s=1), 75 \%(s=5), 80 \%(s=15)$, and $90 \%(s=75)$.

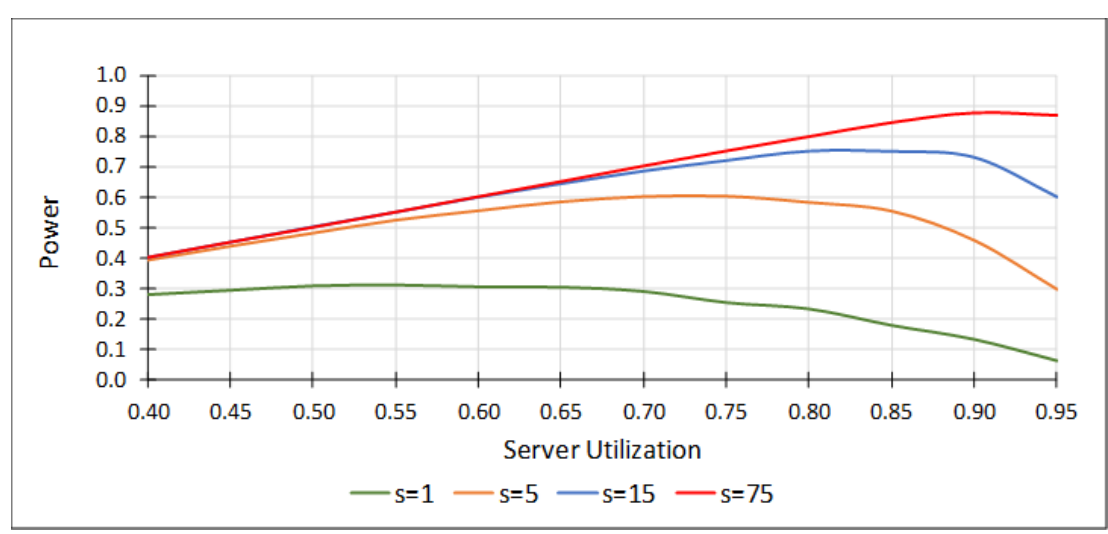

Figure 4: Utilization vs. Power Function (Random arrivals, $\mathrm{CV}=0.5$ ).

An analysis of variance showed that each of the three independent variables $(\mathrm{CV}, s$, and the pattern of arrivals), as well as their two-way interactions, affected the value of the knee (in all cases p-values were 0.001 or less). These relationships are summarized in Figure 5. This result illustrates the value in systems where patients arrive based on appointments, although it is not always possible. It also shows that optimal capacity buffers are differ depending on the amount of service time variation.

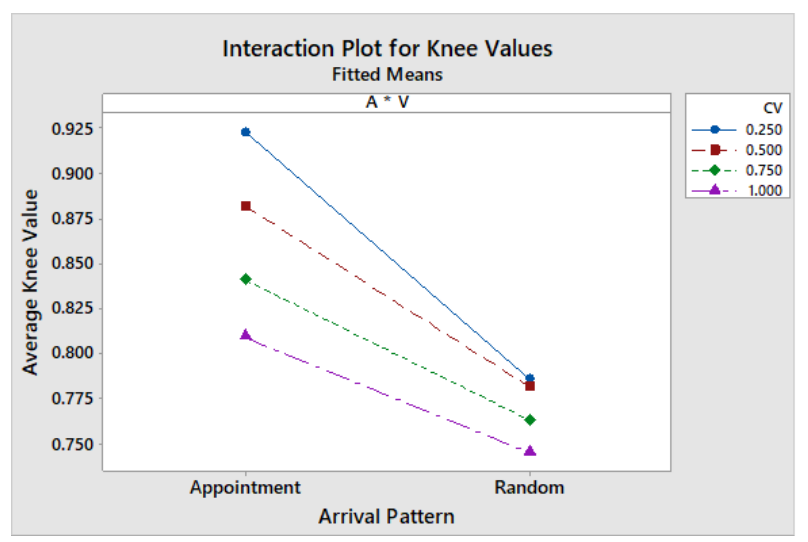

Figure 5: Knee Values versus Arrival Patterns. 


\section{DECISION SUPPORT SYSTEM}

A DSS has been developed using the Python code. The DSS provides a comprehensive tool that evaluates the current capacity planning parameters and shows the optimal server utilization (i.e., the capacity buffer) based on the input parameters provided by the user. Figure 6 shows the user interface, where the user enters the average service rate, average service time, service time $\mathrm{CV}$, the number of servers, and a choice of random or scheduled arrivals. The DSS begins by simulating the queuing system based on the user inputs, then it finds the knee using the approach described earlier. The program then calculates the changes required to move from the current $\rho$ to the optimal $\rho$, either by changing the average service time or by changing the number of servers.

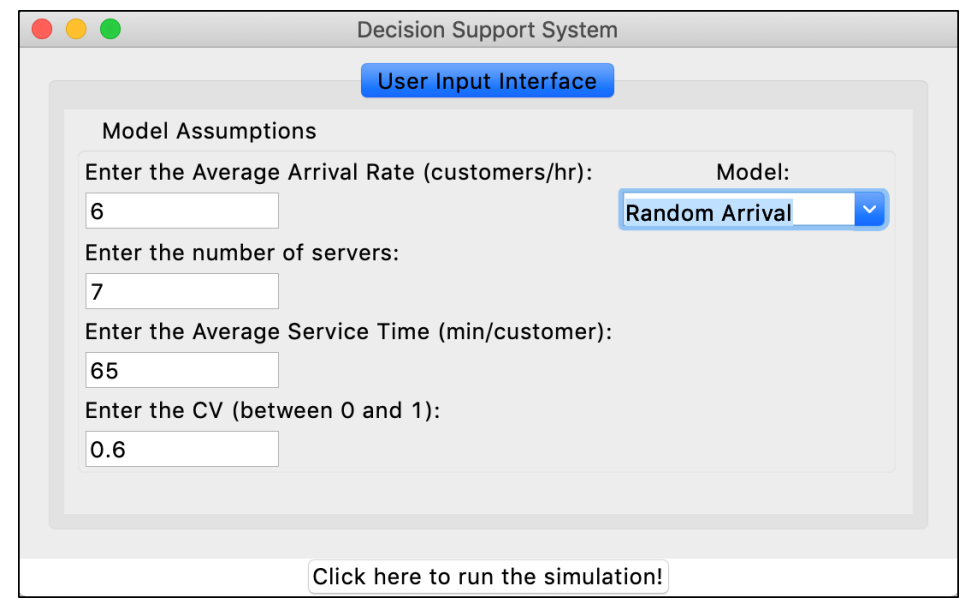

Figure 6: DSS User Interface.

Both tabular and graphical reports are provided as output. Figure 7(a) shows results with the current server utilization compared to results with a range of utilization values based on service time changes. Server utilization is $92.9 \%$ based on an average service time of 65 minutes, which results in an average time in system $\left(W_{s}\right)$ of 135.8 minutes, an average wait time $\left(W_{q}\right)$ of 70.8 minutes, an average patient population $\left(L_{s}\right)$ of 14.1 , and an average queue length $\left(L_{q}\right)$ of 7.1 patients. At the optimal knee $(75 \%$ server utilization) the average service time would be 52.5 minutes, which would result in an average time in system of 60.9 minutes, an average queue time of 8.4 minutes, and average patient population of 6.6, and an average queue length of 0.8 patients. The output shows these results graphically, using the hockey-stick format. Here, we see the current average service (65 minutes) with its corresponding wait time. The knee is located at the average service time of 52.5 minutes. That is, a 12.5 minute decrease in average service time would decrease the average wait for customers by about 62.4 minutes.

Figure 7(b) is an alternative analysis that focuses on changing the number of servers to optimize the configuration. The tabular output shows the results for the current number of servers $(s=7)$ along with various alternatives $(s=8$ and $s=9)$. The graphical output shows that the optimal number of servers is 8.7 (calculated based on the knee of $75 \%$ ). This value is rounded to create the recommendation of 9 servers. The results show that adding two servers reduces the average customer wait time by 66.7 minutes. The decision maker is not required to choose this number of servers. As the graphical output shows, a suitable alternative may be to employ 8 servers, which decreases the average customer wait time by 56.7 minutes. 


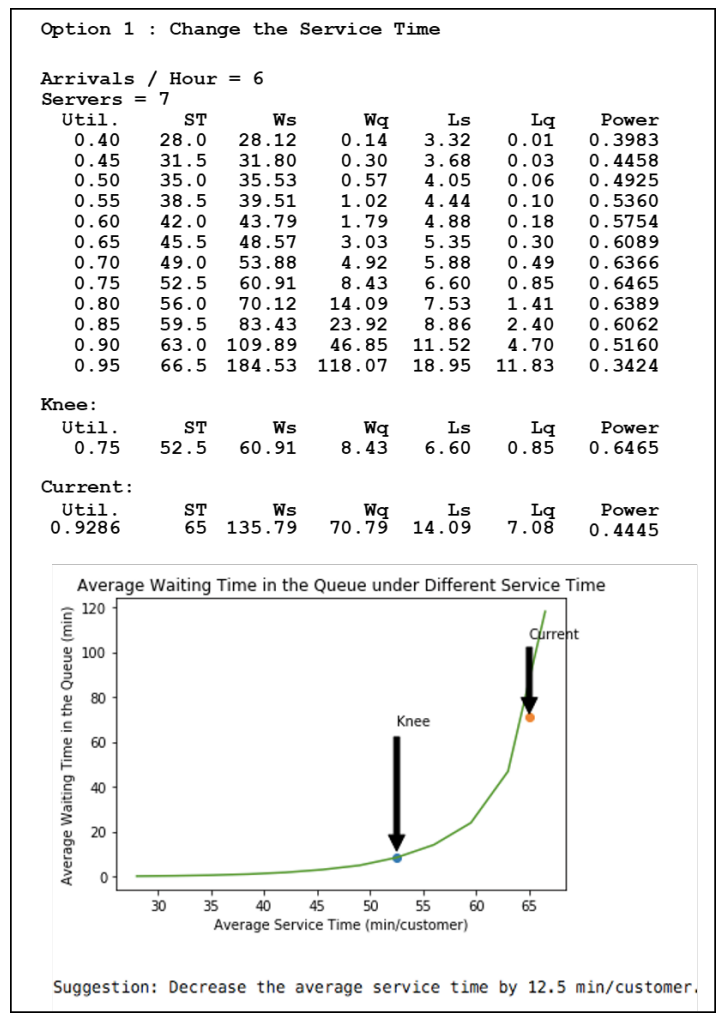

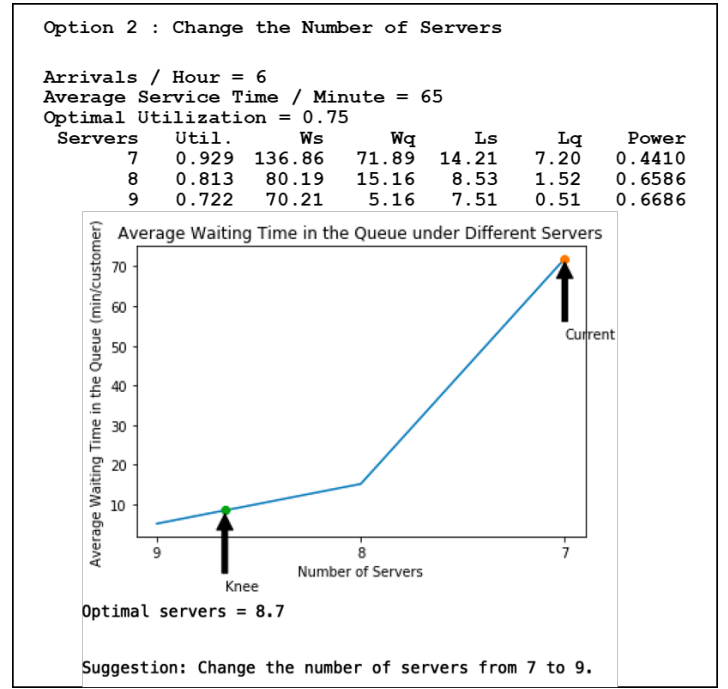

(b)

(a)

Figure 7: (a) Output for Service Time Adjustment. (b) Output for Number of Services Adjustment.

\section{CONCLUSION}

The results of this study show that capacity planning in the presence of uncertainty cannot be done using simple rules of thumb or values that remain constant across situations. In a hospital, planning resource allocations in an ED would require different buffering levels than planning capacity in a small medical office, a large medical practice, or a hospital's call center.

A DSS is provided that is useful for capacity planners to determine suitable configurations for resource-limited facilities. The DSS incorporates simulation and optimization models that make robust real-world assumptions. Unlike simulations of specific facilities, the embedded simulation mimics queues in a general fashion making it applicable to a variety of situations without the need for re-programming.

\section{REFERENCES}

Benneyan, J. C., and F. C. Kaminsky. 1996. "Statistical and economic models for analysis and optimal design of laboratory screening policies for cervical cancer". Annals of Operations Research 67 (1): 235-285.

Berner, E. S., and T. J. La Lande. 2007. "Overview of clinical decision support systems". In Clinical Decision Support Systems, 3-22. Springer.

Bittencourt, O., V. Verter, and M. Yalovsky. 2018. "Hospital capacity management based on the queueing theory". International Journal of Productivity and Performance Management 67 (2): 224-238.

Camacho, F., R. Anderson, A. Safrit, A. S. Jones, and P. Hoffmann. 2006. "The relationship between patient's perceived waiting time and office-based practice satisfaction". North Carolina Medical Journal 67 (6): 409-413.

Deming, W. E. 1986. Out of the Crisis. Cambridge, MA: MIT Center for Advanced Engineering Study.

Fanti, M. P., G. Iacobellis, W. Ukovich, V. Boschian, G. Georgoulas, and C. Stylios. 2015. "A simulation based Decision Support System for logistics management". Journal of Computational Science 10:86-96. 
Gonzalez-Horta, F. A., R. A. Enriquez-Caldera, J. M. Ramirez-Cortes, J. Martínez-Carballido, and E. Buenfil-Alpuche. 2011. "Mathematical model for the optimal utilization percentile in M/M/1 systems: A contribution about knees in performance curves". arXiv:1106.2380.

Grossman, M. 1972. "On the concept of health capital and the demand for health". Journal of Political Economy 80 (2): 223-255.

Hertz, P., S. Cavalieri, G. R. Finke, A. Duchi, and P. Schönsleben. 2014. "A simulation-based decision support system for industrial field service network planning". Simulation 90 (1): 69-84.

Jain, P., and R. Ali. 2016. "A case study of take away restaurant using simulation modelling approach". Journal of Service Science Research 8 (2): 207-221.

Kadri, F., S. Chaabane, and C. Tahon. 2014. "A simulation-based decision support system to prevent and predict strain situations in emergency department systems". Simulation Modelling Practice and Theory 42:32-52.

Kang, S. W., and H. S. Park. 2015. "Emergency department visit volume variability". Clinical and Experimental Emergency Medicine 2 (3): 150.

Kennedy, M. H. 2009. "Simulation modeling for the health care manager". The Health Care Manager 28 (3): $246-252$.

Kleinrock, L. 2018. "Internet congestion control using the power metric: Keep the pipe just full, but no fuller". Ad Hoc Networks 80:142-157.

Koutsoukis, N.-S., and G. Mitra. 2003. Decision Modelling and Information Systems: The Information Value Chain. Springer Science \& Business Media.

Laskowski, M., R. D. McLeod, M. R. Friesen, B. W. Podaima, and A. S. Alfa. 2009. "Models of emergency departments for reducing patient waiting times". PloS One 4 (7): e6127.

Lee, Y., and A. Elcan. 1996. "Simulation modeling for process reengineering in the telecommunications industry". Interfaces 26 (3): 1-9.

Lynn, S. K., and L. F. Barrett. 2014. ““Utilizing” signal detection theory”. Psychological Science 25 (9): $1663-1673$.

Maleyeff, J., L. B. Newell, and F. C. Kaminsky. 2004. "Probability modeling applied to CAD systems for mammography". International Journal of Health Care Quality Assurance 17 (3): 125-134.

Marcial, L. H., J. E. Richardson, B. Lasater, B. Middleton, J. A. Osheroff, K. Kawamoto, J. S. Ancker, D. van Leeuwen, E. A. Lomotan, S. Al-Showk et al. 2018. "The imperative for patient-centered clinical decision support". eGEMs 6 (1).

Millhiser, W. P., and E. A. Veral. 2019. "A decision support system for real-time scheduling of multiple patient classes in outpatient services". Health care management science 22 (1): 180-195.

Nicolaou, L. M. 2016. "Quality improvement and leadership capacity development through lean methodology". The American Journal of Accountable Care 9 (16): 38-43.

Patel, N. M. 2015. "Half-latency rule for finding the knee of the latency curve". ACM SIGMETRICS Performance Evaluation Review 43 (2): 28-29.

Reyna, V. F., W. L. Nelson, P. K. Han, and M. P. Pignone. 2015. "Decision making and cancer.". American Psychologist 70 (2): 105.

Schiff, G. D. 2011. "System dynamics and dysfunctionalities: levers for overcoming emergency department overcrowding". Academic Emergency Medicine 18 (12): 1255-1261.

Schulz, M. 2017. "The intertwined relationship between patient education, hospital waiting times and hospital utilization". Health Services Management Research 30 (4): 213-218.

Spooner, S. A. 2016. "Mathematical foundations of decision support systems". In Clinical Decision Support Systems, 19-43. Springer.

Statista 2016. "Leading challenges for emergency departments in the US as of 2016". https://www.statista. com/statistics/631285/emergency-department-challenges-according-to-survey/. [Online; accessed 28-January-2020].

Sze, D. Y. 1984. "OR practice - a queueing model for telephone operator staffing". Operations Research 32 (2): 229-249.

Terwiesch, C., K. Diwas, and J. M. Kahn. 2011. "Working with capacity limitations: operations management in critical care". Critical Care 15 (4): 308.

Towers, T. J. 2014. "Pressure and performance: buffering capacity and the cyclical impact of accreditation inspections on risk-adjusted mortality”. Journal of Healthcare Management 59 (5): 323-337. 
Vass, H., and Z. K. Szabo. 2015. "Application of queuing model to patient flow in emergency department. Case study". Procedia Economics and Finance 32:479-487.

Wang, J., J. Li, and P. K. Howard. 2013. "A system model of work flow in the patient room of hospital emergency department". Health Care Management Science 16 (4): 341-351.

Yasnoff, W. A., and P. L. Miller. 2003. "Decision support and Expert Systems in public health". In Public Health Informatics and Information Systems, 494-512. Springer.

Yu, H.-H., K.-H. Chang, H.-W. Hsu, and R. Cuckler. 2019. "A Monte Carlo simulation-based decision support system for reliability analysis of Taiwan's power system: Framework and empirical study". Energy 178:252-262.

Zilm, F., K. Culp, and B. Dorney. 2003. "Virtual ambulatory care: computer simulation applications". The Journal of Ambulatory Care Management 26 (1): 7-21.

\section{AUTHOR BIOGRAPHIES}

CANAN G. CORLU is an Assistant Professor in the Administrative Sciences Department of Metropolitan College at Boston University. She holds a PhD in Operations Management from Carnegie Mellon University. Her email address is canan@bu.edu.

JOHN MALEYEFF is an Associate Professor of the Practice in the Administrative Sciences Department of Metropolitan College at Boston University. He holds a PhD in Industrial Engineering \& Operations Research from the University of Massachusetts.

CHENSHU (CHARLOTTE) YANG holds an MS degree in Applied Business Analytics from Boston University.

TIANHUAI (JOSEPHINE) MA holds an MS degree in Applied Business Analytics from Boston University.

YANTING (EMMA) SHEN holds an MS degree in Financial Management from Boston University. 